\title{
Measuring Cable Sheath Currents to Detect Defects in Cable Sheath Connections
}

\author{
A. Khamlichi', M. Adel ${ }^{2}$, F.Garnacho³, J. Rovira \\ LCOE-FFII, Madrid, Spain \\ 1ak@lcoe.etsii.upm.es \\ 2madel@Icoe.etsii.upm.es \\ 3fernandog@lcoe.etsii.upm. \\ es
}

\begin{abstract}
This paper presents a method for detecting different types of defects in the cable sheaths interconnected in a cross bonding configuration of a HV cable system installed in flat or trefoil layout using the sheath currents as input data. Three different defects have been analyzed: electric continuity loss of earth connection, short-circuit between sectionalized metal sheaths of a cable joint and linkbox flooding. The sheath current has been calculated by two different approaches, one on the basis of theoretical equations, programed in MATLAB ${ }^{\circledR}$ and the other on the basis of ATP software simulating the cable system model. The results obtained using both methods have achieved a good agreement between them. A simple criterion by the means of a easy normalized code of four discrete levels $0,1,2$ and 3 has been developed to distinguish the magnitude level of change of sheath currents in case of the indicated defect existence in order to trigger alarm.
\end{abstract}

Index Terms- Hv. Cable; Sheath current, Earth disconnection; ATP software; defect detection in sheaths; earth disconnection; short-circuit in cable joints, link-box flooding; defect alarm.

\section{INTRODUCTION}

Nowadays underground cables are widely used in transmission of electrical energy [1]. However, there are many cases of cable failures that occurred in the network, causing loss of energy, blackouts or serious fires, where not any previous indication of existing defect was detected. In addition, if a defect occurs along a cable system, it is very difficult to locate it by simple visual inspection due to the long length and non-visibility of the cable placed underground. Taking into account that, as the length of the cable increases the induced voltage at cable metal sheaths also increase [2], a greater probability of cable failure appears if additional defect occurs in cable sheaths. As it is well known, to reduce the circulating current through metal cable sheaths, cross bonding connection are used. In reference [3], the failures of cables are classified into 6 groups, namely adverse environmental conditions, third party damage, poor workmanship, manufacturing problems, operational or maintenance reasons and age degradation. According to the statistics made in [4] a good number of cable failures cause excessive sheath current that represents the third party of damages in cable jackets and the breakdown of the insulation between cable sheaths. The feasibility to detect of a cable sheath defect by monitoring sheath currents to ground at the end of the cross-bonded section is presented in [5].

This paper is dedicated to show the change in the sheath current in case of different types of defects and different cable layouts: flat or three phase trefoil, by measuring the current of metal cable sheaths at fixed points corresponding t linkboxes. The sheath currents have been calculated in the normal condition (no defects) by two methods: theoretical equations using the MATLAB $\AA$, and circuit simulation using ATP. Moreover the fault condition has been studied using ATP software and LCOE software.

\section{THEORETICAL MODEL}

Cross bonding configuration consists of 3 minor sections of slightly different lengths (L1, L2 and L3) due to the practical use. Each minor section consists of 3 phase cables of equal lengths. The metal cable sheaths of each phase cable are connected to the earth at both cable terminations. A link box is used to connect the minor sections to each other to for transposition purposes. Figure 1 shows the typical model of the cross bonding sheath connection while figure 2 shows the simplified circuit of a cross bonding sheath connection indicating the circulating currents pass through the three loops of interconnected metal sheaths $\left(J_{l 1}, J_{l 2}, J_{l 3}\right)$ and the load currents pass through 3 phase conductors $\left(J_{1}, J_{2}, J_{3}\right)$. The sheath currents pass through three different loops of cable sheaths: loop1-red one (Loop 1, $\left.J_{l 1}\right)$, loop2-blue one(loop2, $J_{l 2}$ ) and loop 3-green one(Loop3, $J_{l 3}$ ), as it is shown in figure 2 . As stated in [6] sheath current in each loop of cable sheath is composed of leakage current through the main insulation and the circulating current due to the unbalanced induced voltage in each cable sheath loop.

\section{Induced currents in cable sheaths due to magnetic coupling}

The induced voltage in each sheath loop is caused by both the currents: the currents of the phase conductors and the currents of the metal cable sheaths. 


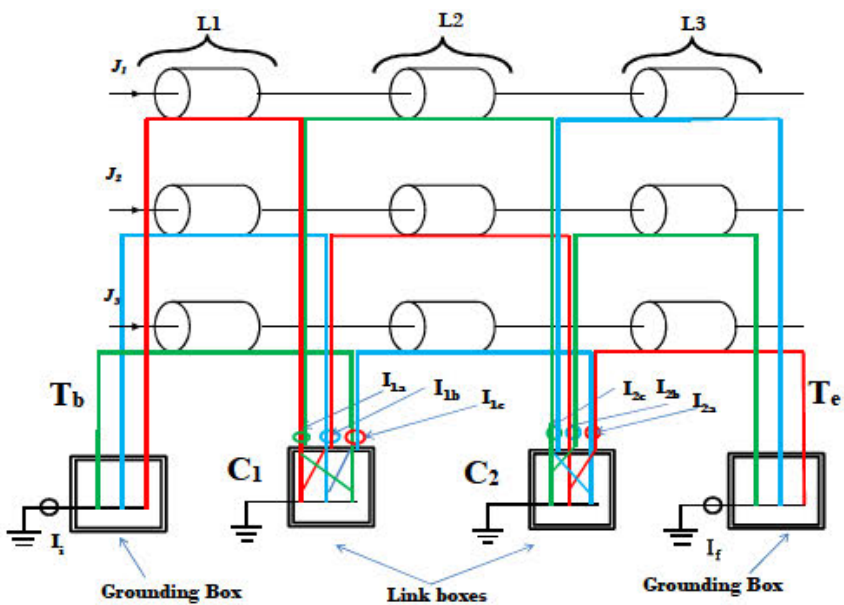

Figure 1: Cross bonding configuration of a high voltage cable system

The load currents through the phase conductors $\left(J_{1}, J_{2}\right.$, and $\left.J_{3}\right)$ of the cable systems induce a voltage in each cable section $L_{i}$ $(i=l$ to 3$)$, of each sheath loop $(1 l, 2 l$ and $3 l$ ) that are given by the following set of equations:

$$
\begin{aligned}
& U_{1 c, L 1}=X_{c-s} \cdot L_{1} \cdot J_{1}+X_{s 1-s 2} \cdot L_{1} \cdot J_{2}+X_{s 3-s 1} \cdot L_{1} \cdot J_{3} \\
& U_{1 c, L 2}=X_{s 1-s 2} \cdot L_{2} \cdot J_{1}+X_{c-s} \cdot L_{2} \cdot J_{2}+X_{s 2-s 3} \cdot L_{2} \cdot J_{3} \\
& U_{1 c, L 3}=X_{s 3-s 1} \cdot L_{3} \cdot J_{1}+X_{s 2-s 3} \cdot L_{3} \cdot J_{2}+X_{c-s} \cdot L_{3} \cdot J_{3} \\
& U_{2 c, L 1}=X_{s 1-s 2} \cdot L_{1} \cdot J_{1}+X_{c-s} \cdot L_{1} \cdot J_{2}+X_{s 2-s 3} \cdot L_{1} \cdot J_{3} \\
& U_{2 c, L 2}=X_{s 3-s 1} \cdot L_{2} \cdot J_{1}+X_{s 2-s 3} \cdot L_{2} \cdot J_{2}+X_{c-s} \cdot L_{2} \cdot J_{3} \\
& U_{2 c, L 3}=X_{c-s} \cdot L_{3} \cdot J_{1}+X_{s 1-s 2} \cdot L_{3} \cdot J_{2}+X_{s 3-s 1} \cdot L_{3} \cdot J_{3} \\
& U_{3 c, L 1}=X_{s 3-s 1} \cdot L_{1} \cdot J_{1}+X_{s 2-s 3} \cdot L_{1} \cdot J_{2}+X_{c-s} \cdot L_{1} \cdot J_{3} \\
& U_{3 c, L 2}=X_{c-p} \cdot L_{2} \cdot J_{1}+X_{s 1-s 2} \cdot L_{2} \cdot J_{2}+X_{s 1-s 3} \cdot L_{2} \cdot J_{3} \\
& U_{3 c, L 3}=X_{s 1-s 2} \cdot L_{3} \cdot J_{1}+X_{c-s} \cdot L_{3} \cdot J_{2}+X_{s 2-s 3} \cdot L_{3} \cdot J_{3}
\end{aligned}
$$

where:

$U_{i c-L j}$ is the induced voltage by the currents of the three phase conductors " $c$ " in the loop $i$ corresponding to the cable section $j$ of length $L_{j}$

$X_{c-s}$ is the mutual inductance between cable conductor and its cable sheath per length unit.

$X_{s i-5 j}$ is the mutual inductance between the cable sheath $i$ and the cable sheath $j$ per length unit.

$$
\begin{aligned}
& X_{c-s}=\frac{\omega \cdot \mu_{o}}{2 . \pi} \ln \left(\frac{D_{e}}{r_{s}}\right) \\
& X_{s i-s j}=\frac{\omega \cdot \mu_{o}}{2 . \pi} \ln \left(\frac{D_{e}}{S_{i-j}}\right) \\
& D_{e}=1.85 \sqrt{\frac{100}{\omega \pi}}
\end{aligned}
$$

where,

$D_{e}$ is the equivalent distance to earth. $S_{i-j}$ is the distance between phases $\mathbf{i}, \mathbf{j}$. $r_{s}$ is the radius of the sheath.

As it is shown in figure 2, the sum of induced voltages along of each loop of cable sheaths (loop1-red one, loop2-blue one and loop 3-green one) caused by the currents phase conductors $\left(J_{1}, J_{2}\right.$, and $\left.J_{3}\right)$ are given by the following expressions:

$$
\left.\begin{array}{l}
U_{1 l}=U_{1 l, L 1}+U_{1 l, L 2}+U_{1 l, L 3} \\
U_{2 l}=U_{2 l, L 1}+U_{2 l, L 2}+U_{2 l, L 3} \\
U_{3 l}=U_{3 l, L 1}+U_{3 l, L 2}+U_{3 l, L 3}
\end{array}\right]
$$

Similarly, the currents through the metal cable sheaths ( $J_{l l}$, $J_{I 2}$, and $J_{13}$ ) of the cable system induce a voltage in the cable section $L_{i}(i=l$ to 3$)$ of each sheath loop $(1 l, 2 l$ and $3 l)$ that are given by the following set of equations:

$$
\begin{aligned}
& U_{1 s, L 1}=Z_{s} \cdot L_{1} \cdot J_{l 1}+X_{s 1-s 2} \cdot L_{1} \cdot J_{l 2}+X_{s 3-s 1} \cdot L_{1} \cdot J_{l 3} \\
& U_{1 s, L 2}=Z_{s} \cdot L_{2} \cdot J_{l 1}+X_{s 2-s 3} \cdot L_{2} \cdot J_{l 2}+X_{s 1-s 2} \cdot L_{2} \cdot J_{l 3} \\
& U_{1 s, L 3}=Z_{s} \cdot L_{3} \cdot J_{l 1}+X_{s 3-s 1} \cdot L_{3} \cdot J_{l 2}+X_{s 2-s 3} \cdot L_{3} \cdot J_{l 3} \\
& U_{2 s, L 1}=X_{s 1-s 2} \cdot L_{1} \cdot J_{l 1}+Z_{s} \cdot L_{1} \cdot J_{l 2}+X_{s 2-s 3} \cdot L_{1} \cdot J_{l 3} \\
& U_{2 s, L 2}=X_{s 2-s 3} \cdot L_{2} \cdot J_{l 1}+Z_{s} \cdot L_{2} \cdot J_{l 2}+X_{s 3-s 1} \cdot L_{2} \cdot J_{l 3} \\
& U_{2 s, L 3}=X_{s 3-s 1} \cdot L_{3} \cdot J_{l 1}+Z_{s} \cdot L_{3} \cdot J_{l 2}+X_{s 1-s 2} \cdot L_{3} \cdot J_{l 3} \\
& U_{3 s, L 1}=X_{s 3-s 1} \cdot L_{1} \cdot J_{l 1}+X_{s 2-s 3} \cdot L_{1} \cdot J_{l 2}+Z_{s} \cdot L_{1} \cdot J_{l 3} \\
& U_{3 s, L 2}=X_{s 1-s 2} \cdot L_{2} \cdot J_{l 1}+X_{s 3-s 1} \cdot L_{2} \cdot J_{l 2}+Z_{s} \cdot L_{2} \cdot J_{l 3} \\
& U_{3 s, L 3}=X_{s 2-s 3} \cdot L_{3} \cdot J_{l 1}+X_{s 1-s 2} \cdot L_{3} \cdot J_{l 2}+Z_{s} \cdot L_{3} \cdot J_{l 3}
\end{aligned}
$$

where:

$U_{i s-L j}$ is the induced voltage by the currents of the three metal cable sheaths " $s$ " in the loop $i$ corresponding to the cable section $j$ of length $L_{j}$

$Z_{s}$ is the cable sheath impedance per length unit.

$R_{s} \quad$ is the resistivity of the sheath.

$$
Z_{s}=R_{s}+\frac{\omega \mu_{o}}{8}+\frac{j \omega \mu_{o}}{2 . \pi} \ln \left(\frac{D_{e}}{r_{s}}\right)
$$

The induced voltages along the each loop of cable sheaths (loop1-red one, loop2-blue one and loop 3-green one) caused by the currents of cable sheaths $\left(J_{l l}, J_{l 2}\right.$, and $\left.J_{l 3}\right)$ are given by the following expressions:

$$
\left.\begin{array}{l}
U_{1 s}=U_{1 s, L 1}+U_{1 s, L 2}+U_{1 s, L 3} \\
U_{2 s}=U_{2 s, L 1}+U_{2 s, L 2}+U_{2 s, L 3} \\
U_{3 s}=U_{3 s, L 1}+U_{3 s, L 2}+U_{3 s, L 3}
\end{array}\right]
$$


The total induced voltage in each loop of cable sheaths ( $\mathrm{i}=1$, 2, 3 loops), is given by the superposition of (5) and (8):

$$
\left.\begin{array}{l}
U=U_{1 c}+U_{1 s} \\
U=U_{2 c}+U_{2 s} \\
U=U_{3 c}+U_{3 s}
\end{array}\right\}
$$

In addition, the following boundary restriction must be satisfied

$$
U=\left(R_{1}+R_{2}\right) \cdot\left(J_{l 1}+J_{l 2}+J_{l 3}\right)
$$

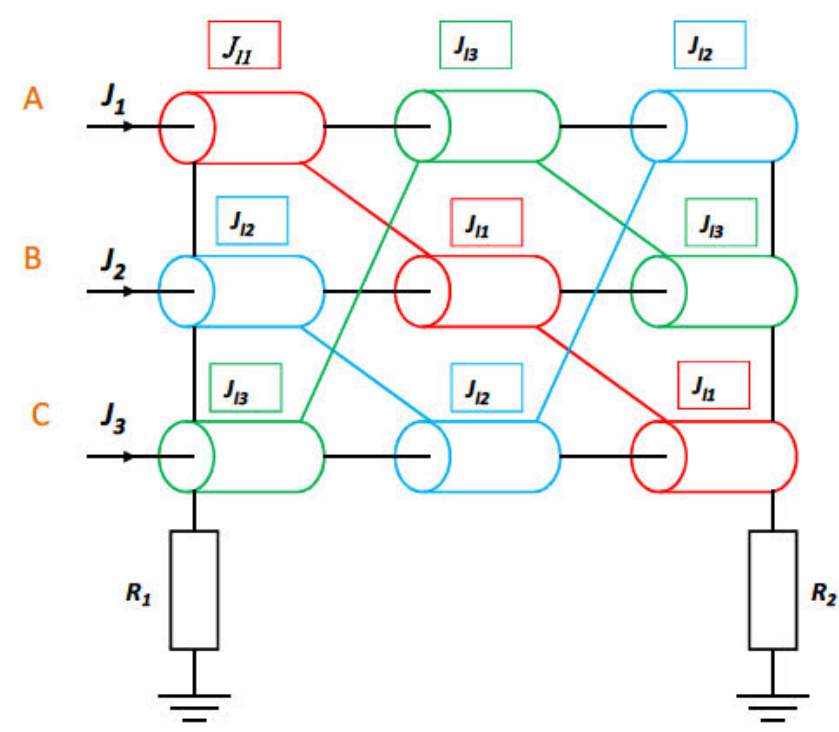

Figure 2:Simplified cross bonding configration.

The induced currents in cable sheaths due capacitive coupling are calculated using the same method stated in [6]. The currents due to magnetic coupling $\left(\mathrm{J}_{11 \mathrm{~m}}, \mathrm{~J}_{12 \mathrm{~m}}, \mathrm{~J}_{13 \mathrm{~m}}\right)$ remain constant along each loop while the capacitive currents change depending on the length of the section. The capacitive currents in the first cross are represented as $\mathrm{J}_{11 \mathrm{c} 1}, \mathrm{~J}_{12 \mathrm{c} 1}, \mathrm{~J}_{13 \mathrm{c} 1}$ and the ones in the second cross are represented as $\mathrm{J}_{11 \mathrm{c} 2}, \mathrm{~J}_{12 \mathrm{c} 2}$, $\mathrm{J}_{13 \mathrm{c} 2}$

The current through each loop is maintained by the means of the super position of the capacitive currents and magnetic currents as dedicated in the following equations.

$$
\begin{aligned}
& J_{L 1 c 1} *=J_{l 1 m}+J_{l 1 c 1} \quad J_{L 1 c 2} *=J_{l 1 m}+J_{l 1 c 2} \\
& J_{L 2 c 1} *=J_{l 2 m}+J_{l 2 c 1} J_{L 2 c 2} *=J_{l 2 m}+J_{l 2 c 2} \\
& J_{L 3 c 1} *=J_{l 3 m}+J_{l 3 c 1} \quad J_{L 3 c 2} *=J_{l 3 m}+J_{l 3 c 2}
\end{aligned}
$$

\section{III.}

\section{CABLE SYSTEM ANALYSIS AND SENSOR LOCATION}

The sheath currents have been analyzed in the normal operation (no defect) and in case of existence of different types of defects investigated on two different types of cable layouts: three phase center-symmetric and flat configuration. A cable system of $220 \mathrm{kV}$ is considered for this analysis which parameters are illustrated in Table 1.

Table 1: Cable parameters

\begin{tabular}{lc}
\hline Parameters & Value \\
\hline Raduis of the conductor $(\mathrm{mm})$ & 55.2 \\
Raduis of insulation $(\mathrm{mm})$ & 51.9 \\
Relative permativity of insulation & 2.5 \\
Exterior sheath diameter $(\mathrm{mm})$ & 114.5 \\
Interior sheath diameter $(\mathrm{mm})$ & 109.68 \\
Sheath resistivity $\Omega \cdot \mathrm{m}$ & $7.2034 .10^{-8}$ \\
Ground resistance $\Omega$ & 0.2 \\
$\mathrm{~S}(\mathrm{~mm})$ & 42.5 \\
$\mathrm{~L}_{1}(\mathrm{~m})$ & 540 \\
$\mathrm{~L}_{2}(\mathrm{~m})$ & 600 \\
$\mathrm{~L}_{3}(\mathrm{~m})$ & 660 \\
Load current for the simulations (A) & 1000
\end{tabular}

Three current sensors fastened around the concentric cables of the exit of cable joints as shown in figurel are considered to measure the differential currents: $I_{1 a}, I_{1 b}, I_{1 c}, I_{2 a}, I_{2 b}$ and $I_{2 c}$ corresponding to the difference of sheath currents between each two loops of each cable sheaths. The currents $I_{I a}, I_{I b}$ and $I_{I c}$ are picked up just before the cross $\mathrm{C}_{1}$ and the currents $I_{2 a}$, $I_{2 b}$ and $I_{2 c}$ just before cross $\mathrm{C}_{2}$ (see figure 1 ).

$$
\begin{array}{ll}
I_{1 a}=J_{L 1 c 1} *-J_{L 3 c 1} * & I_{2 a}=J_{L 3 c 2} *-J_{L 2 c 2} * \\
I_{1 b}=J_{L 2 c 1} *-J_{L 1 c 1} * & I_{2 b}=J_{L 1 c 2} *-J_{L 1 c 1} * \\
I_{1 c}=J_{L 3 c 1} *-J_{L 2 c 1} * & I_{2 c}=J_{L 2 c 2} *-J_{L 1 c 2} *
\end{array}
$$

Two different approaches have been used to calculate the sheath current in normal case (no defect). The first approach is based on calculating the sheath current using the theoretical equations from (1)-(12). This approach has been implemented using MATLAB $B$ tool. The second approach is calculating sheath currents using ATP software. Figure 3 shows the implemented cable model in ATP software with three sections $L_{1}(540 \mathrm{~m}), L_{2}(600 \mathrm{~m})$ and $L_{3}(660 \mathrm{~m})$ and two sheath crosses $C_{1}$ and $C_{2}$ where current sensor will be placed to pick up the data $I_{1 a}, I_{1 b}, I_{1 c}, I_{2 a}, I_{2 b}$ and $I_{2 c}$. The cable system has been implemented like an transmission line with its equivalent $\Pi$-circuit model. The resistances marked by " $\mathrm{f}$ " with a very small value, are used only to connect the 3-phase cable to the grounding resistances, R1 and R2, whose values are $0.2 \Omega$ and $0.2 \Omega$. Figures 4 and 5 show the cable layout for both three phace center-symmetric and flat layouts. Table 1 shows the obtained results for the normal opration (no defect) calculated by both ATP software and by formula

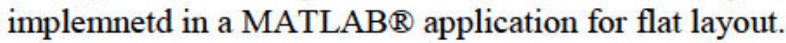


Table 1: Simulated and calculated currents in sensors.

\begin{tabular}{|c|c|c|c|c|c|c|c|}
\hline \multicolumn{2}{|c|}{ Differential Currents } & $I_{I a}$ & $I_{l b}$ & $I_{l c}$ & $I_{2 a}$ & $I_{2 b}$ & $I_{2 c}$ \\
\hline \multirow{3}{*}{ Flat layout } & ATP modelling & 935 & 909 & 642 & 714 & 1001 & 1001 \\
\cline { 2 - 9 } & Matlab equations & 932 & 903 & 639 & 728 & 1012 & 1001 \\
\hline
\end{tabular}

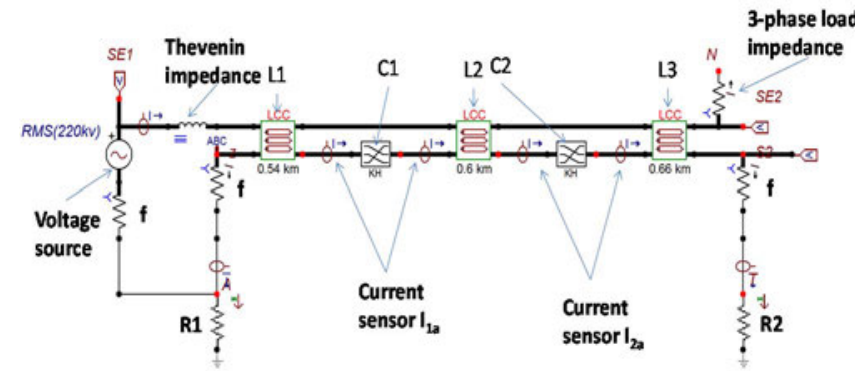

Figure 3: ATP model of the cable system under analysis.
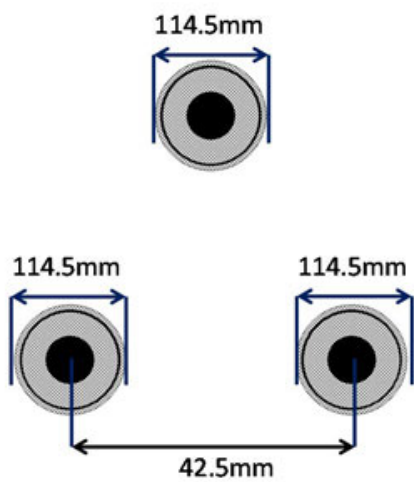

Figure 4: Trefoil layout

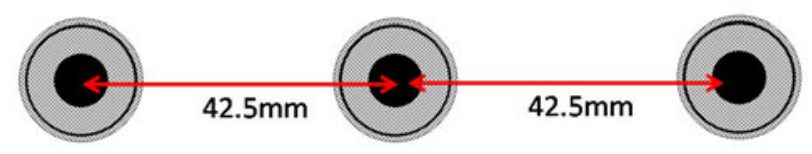

Figure 5: Flat layout

\section{DETECTION CRITERIA}

The results of each sensor are expressed in its per-unit value (p.u) related to its base value chosen as the current value in the normal operation (without defect). Moreover, the values obtained are referred in four discrete levels, $0,1,2$ and 3 following the following criterion (see figure 4):

- If $I_{i j} \leq 0.75 p u$ Level=0 below normal value.

- If $0.75 p u<I_{i j} \leq 1.25 p u \quad$ Level=1 normal value.

- If $1.25 p u<I_{i j} \leq 7.5 p u \quad$ Level= 2 up normal value.

- If $I_{i j}>7.5 p u \quad$ Level=3 very above normal value.
By this way, a general code of 6 digits is used to detect the type of the defect that is produced in the cable system that has one cross bonding configuration. When the code of 6 digits is different to 111111 some defect is happening assuming that a discrepancy larger $25 \%$ between real measured differential currents and the calculated ones are caused which couldn't be justified due to measuring, modelling uncertainties.

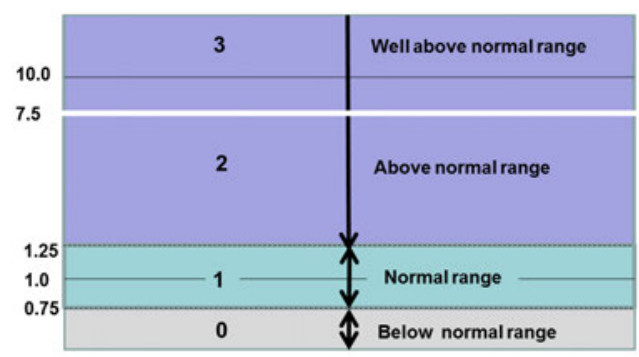

Figure 6 Criterion to normalize the results.

\section{ANALYISIS OF RESULTS}

This paper presents an analysis of 3 types of defects: a) electrical continuity loss of any cable sheath loop, b) linkboxes flooding and c) short-circuit between sectionalized sheaths of a joint, by calculating the sheath currents by ATP software.

\section{V.1 Defect 1: electrical continuity loss}

The defect corresponding to an electric continuity loss of cable sheaths has been implemented in ATP software by intercalating an infinite resistance along each loop of cable sheaths at four different sites: at the beginning terminal $\mathrm{T}_{\mathrm{b}}$, at the first cross $C_{1}$, at the second cross $C_{2}$ and at the end terminal $T_{e}$ (see figure 2).

The analyzed results are obtained from the set of 6 sensors $\left(I_{l a}, I_{l b}, I_{1 c}, I_{2 a}, I_{2 b}, I_{2 c}\right)$ located in both crosses of sheathes, C1 and $\mathrm{C} 2$ (see figure 1).

\section{Case1: Cross bonding in trefoil layout}

Tables 2 and 3 show that when an electrical continuity is lost in a loop the p.u. values obtained from the six sensors are very close to each other with a low influence where the disconnection occurred along the loop, (in $\mathrm{T}_{\mathrm{i}}, \mathrm{C}_{1}, \mathrm{C}_{2}$ or $\mathrm{T}_{\mathrm{f}}$ ). From this p.u. values, the resulting normalized codes indicate the type of the defect as shown in table 2.

By applying the same analysis on loop2 and loop3, it is found that the results are analogous to the ones obtained from loop1 which means that there is not significant dependency of the site where the electrical continuity is lost on the measured differential currents $I_{1 a}, I_{1 b}, I_{1 c}, I_{2 a}, I_{2 b}$ and $I_{2 c}$. In conclusion the site where the disconnection happens does not affect the results. However the code gives different results when the defect is in other loops as it is shown in Table 4. 
Table 4: Codes due to the loss of electrical continuity in sheath loops

Table 2: Continuity loss in loop1 of metal sheaths in trefoil layout

\begin{tabular}{|l|c|c|c|c|c|c|}
\hline $\begin{array}{c}\text { Site of the } \\
\text { continuity } \\
\begin{array}{c}\text { loss } \\
\text { along loop1 }\end{array}\end{array}$ & $\begin{array}{c}\mathrm{I}_{1 \mathrm{a}} \\
(\mathrm{pu})\end{array}$ & $\begin{array}{c}\mathrm{I}_{1 \mathrm{~b}} \\
(\mathrm{pu})\end{array}$ & $\begin{array}{c}\mathrm{I}_{1 \mathrm{c}} \\
(\mathrm{pu})\end{array}$ & $\begin{array}{c}\mathrm{I}_{2 \mathrm{a}} \\
(\mathrm{pu})\end{array}$ & $\begin{array}{c}\mathrm{I}_{2 \mathrm{~b}} \\
(\mathrm{pu})\end{array}$ & $\begin{array}{c}\mathrm{I}_{2 \mathrm{c}} \\
(\mathrm{pu})\end{array}$ \\
\hline Terminal $\mathrm{T}_{\mathrm{b}}$ & 0.4 & 0.6 & 1.0 & 1.0 & 0.5 & 0.5 \\
\hline Cross $\mathrm{C}_{1}$ & 0.5 & 0.5 & 1.0 & 1.0 & 0.6 & 0.5 \\
\hline Cross $\mathrm{C}_{2}$ & 0.4 & 0.6 & 1.0 & 1.0 & 0.5 & 0.5 \\
\hline Terminal $\mathrm{T}_{\mathrm{e}}$ & 0.4 & 0.6 & 1.0 & 1.0 & 0.5 & 0.5 \\
\hline Code & $\mathbf{0}$ & $\mathbf{0}$ & $\mathbf{1}$ & $\mathbf{1}$ & $\mathbf{0}$ & $\mathbf{0}$ \\
\hline
\end{tabular}

\section{Case2: Cross bonding in flat layout}

The same analysis of case one is applied when the cable system is installed with the flat layout shown in figure 5 . Table 3 illustrates the results obtained in p.u values when the electrical continuity is lost in loop1. Similarly to the case 1, it can also be noted that the results are almost similar regardless of the branch in which the loss of electrical continuity occurs along the loop 1 .

Table 3 Continuity loss in loop 1 of metal sheaths in flat layout

\begin{tabular}{|c|c|c|c|c|c|c|}
\hline $\begin{array}{c}\text { Site of the continuity loss } \\
\text { along loop1 }\end{array}$ & $I_{l a}$ & $I_{l b}$ & $I_{l c}$ & $I_{2 a}$ & $I_{2 b}$ & $I_{2 c}$ \\
\hline Terminal $\mathrm{T}_{\mathrm{b}}$ & 0.3 & 0.3 & 1.0 & 1.0 & 0.5 & 0.3 \\
\hline Cross $\mathrm{C}_{1}$ & 0.4 & 0.2 & 1.0 & 1.0 & 0.5 & 0.2 \\
\hline Cross $\mathrm{C}_{2}$ & 0.4 & 0.3 & 1.0 & 1.0 & 0.5 & 0.3 \\
\hline Terminal $\mathrm{T}_{\mathrm{e}}$ & 0.3 & 0.3 & 1.0 & 1.0 & 0.4 & 0.3 \\
\hline Code & $\mathbf{0}$ & $\mathbf{0}$ & $\mathbf{1}$ & $\mathbf{1}$ & $\mathbf{0}$ & $\mathbf{0}$ \\
\hline
\end{tabular}

Table 4 illustrates the normalization codes in trefoil and flat layouts in case of loss of electrical continuity in loop 1 or 2 or3. The resulting codes are very different when the continuity loss occurs in a different loop with no dependency on layout used.

\section{V.2 Defect 2: Flooding in link-boxes}

Flooding in link-boxes, where the connection of sheaths is performed, leads to short circuit simultaneously in the 3 cable sheaths where the flooding occurs. This defect has been implemented in ATP by short-circuiting the 3 phased sheaths at the corresponding link-box.

As a result of that, significant increasing in the differential currents occurs independently of what cable layout is used either with trefoil or with flat layout. So in this case the normalization code will be well above normal range for every sensor: 333333 .

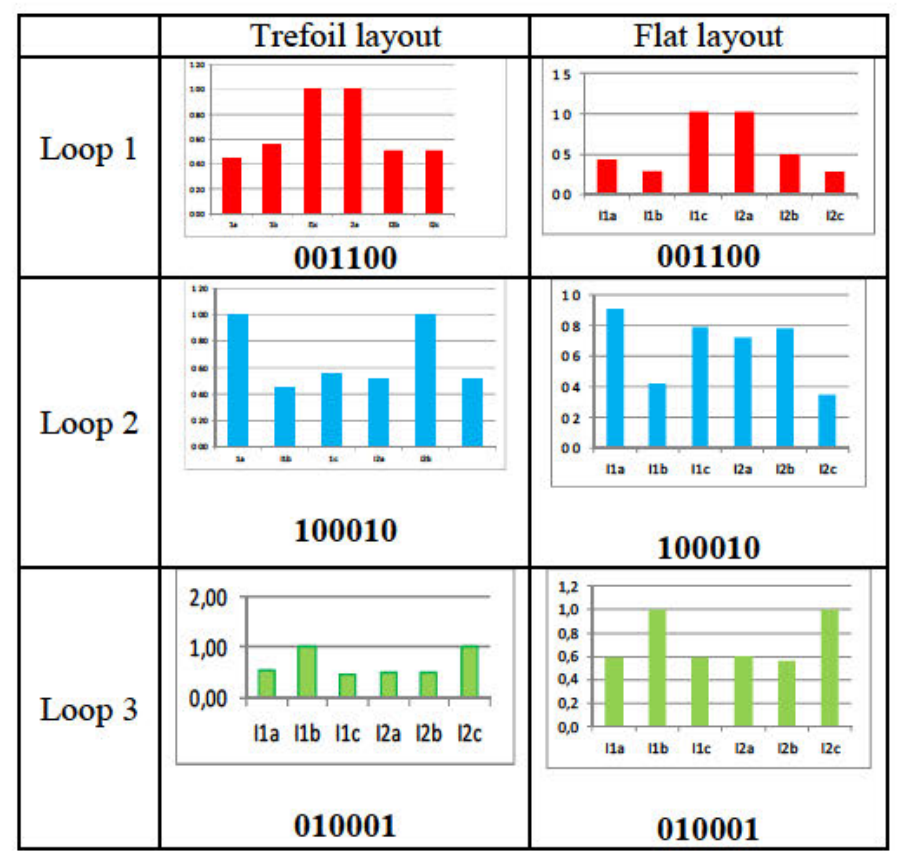

Table 5: Flooding defect analysis in flat layout

\begin{tabular}{|c|c|c|c|c|c|c|}
\hline Flooding location & $I_{1 a}$ & $I_{1 b}$ & $I_{l c}$ & $I_{2 a}$ & $I_{2 b}$ & $I_{2 c}$ \\
\hline $\mathrm{C}_{1}$ & 9.3 & 9.3 & 9.3 & 8.2 & 8.2 & 8.2 \\
\hline $\mathrm{C}_{2}$ & 8.9 & 8.9 & 8.9 & 7.8 & 7.8 & 7.8 \\
\hline Code & $\mathbf{3}$ & $\mathbf{3}$ & $\mathbf{3}$ & $\mathbf{3}$ & $\mathbf{3}$ & $\mathbf{3}$ \\
\hline $\mathrm{C}_{1}$ and $\mathrm{C}_{2}$ & 0.04 & 0.04 & 0.04 & 0.04 & 0.04 & 0.04 \\
\hline Code & $\mathbf{0}$ & $\mathbf{0}$ & $\mathbf{0}$ & $\mathbf{0}$ & $\mathbf{0}$ & $\mathbf{0}$ \\
\hline
\end{tabular}

Table 6: Flooding defect analysis

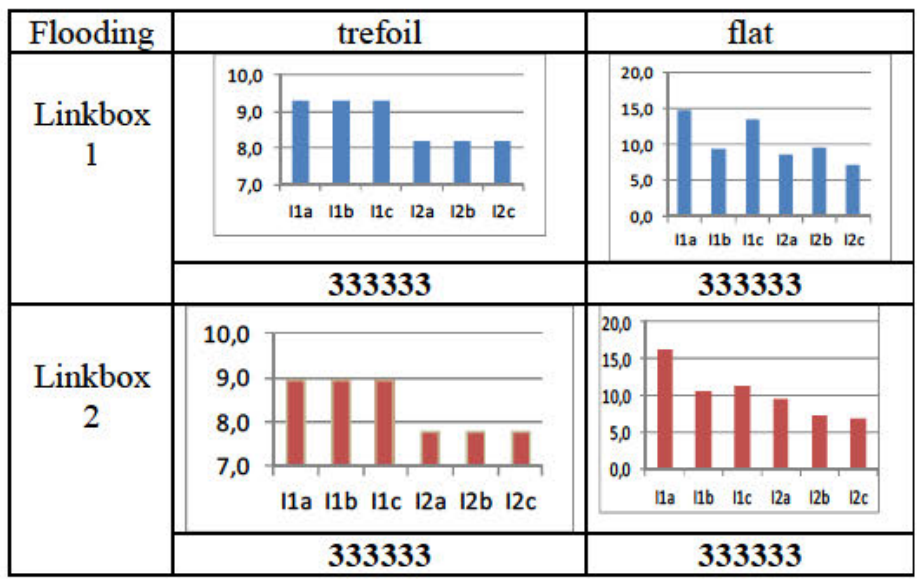

Table 5 shows the obtained results in p.u. when the flooding occurs with a flat layout. Table 6 illustrates the summary of the obtained results and associated codes for both trefoil and flat layout. 


\section{V.3 Defect 3: Short-circuit between sectionalized sheaths}

Short-circuit between sectionalized metal sheaths of a cable joint leads to increase differential currents in some sensors more than in others. Table 7 shows the obtained results when the cable is installed with both layouts: trefoil and flat layout, together their associated normalized defect codes. This kind of defect has been also implemented in ATP software by interconnecting a very small resistance between the metal sheathes coming from the same cable joint.

Table 7. Short-circuit between sectionalized sheaths

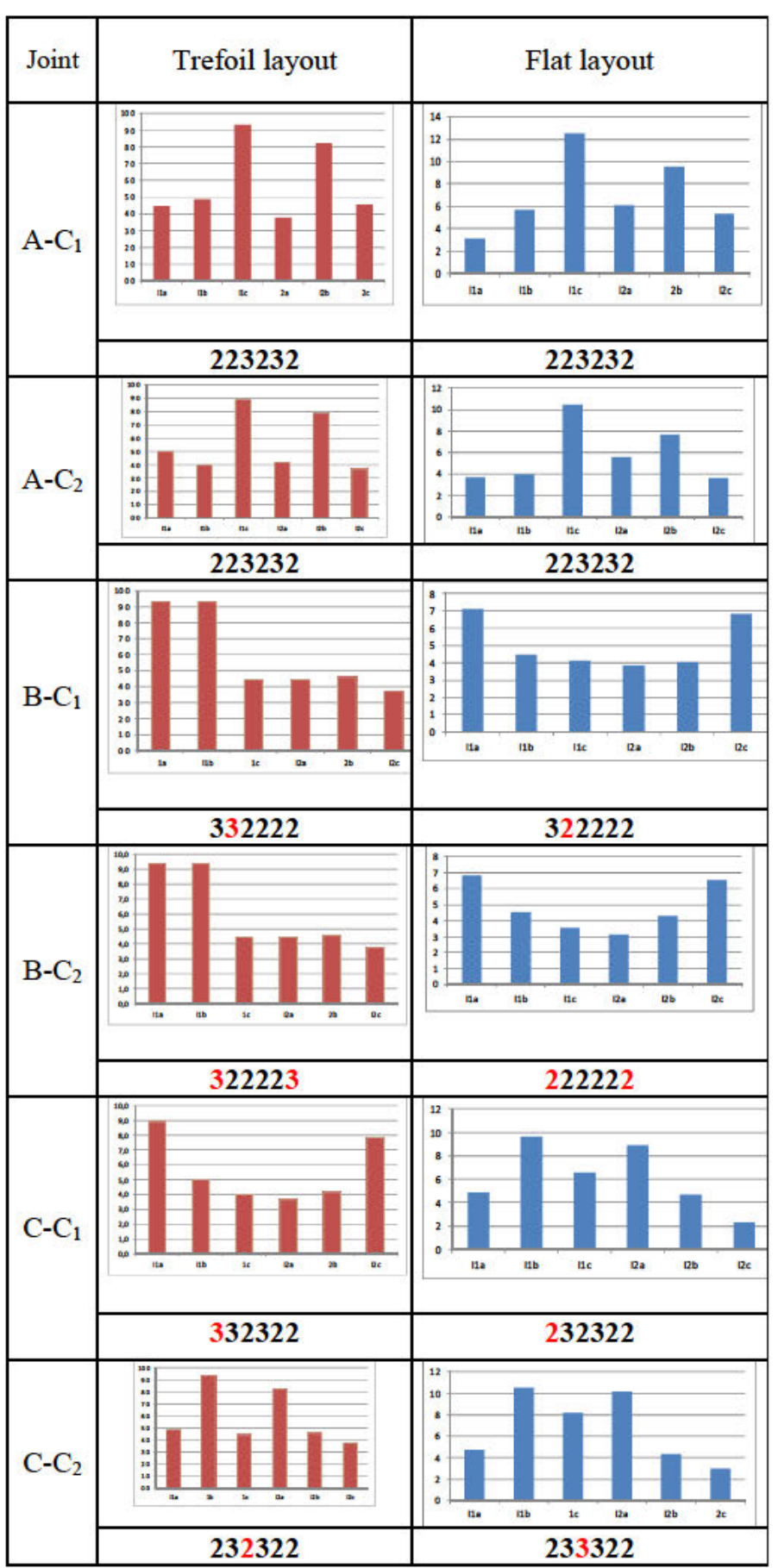

Similarly to flooding defect, here it can be also noted that the resulting codes are very different when short-circuit occurs in a different joint (A, B or C) and they are very similar in both layouts. Furthermore there is not significant influence on the cross where the defect occurs $\left(\mathrm{C}_{1}\right.$ or $\left.\mathrm{C}_{2}\right)$.

\section{CONCLUSIONS}

This paper illustrates a method to detect three different types of defects in metal sheaths of HV cable systems: a) electrical continuity loss of the metal sheaths, b) flooding of link-box and short-circuit of sectionalized sheaths of a cable joint. A cable system of $220 \mathrm{kV}$ installed in a cross bonding configuration with flat and trefoil layouts were used to analyze the differential currents measured in concentric cables used to connect each accessories with link-boxes. Six currents sensors are fastened on concentric cables to acquire the input data. The results have demonstrated that the three different defects can be detected and distinguished if the measured currents are compared with the expected values in normal operation (no defect) and simple magnitude criterion is used to obtain a defect code of six digits.

\section{ACKNOWLEDGEMENT}

This work has received funding from the European Union's Horizon 2020 research and innovation programme under the Marie Sklodowska-Curie grant agreement No 676042 .

\section{REFRENCES}

[1] J. Song-Manguelle, M. Harfman Todorovic, S. Chi, S. Gunturi and R. Datta, "Power Transfer Capability of HVAC Cables for Subsea Transmission and Distribution Systems", IEEE Transactions on Industry Applications, vol. 50, no. 4, pp. 2382-2391, 2014.

[2] M. D'Amore, M. Sarto and A. Scarlatti, "Modeling of magnetic-field coupling with cable bundle harnesses", IEEE Transactions on Electromagnetic Compatibility, vol. 45, no. 3, pp. 520-530, 2003.

[3] Z. Tang, C. Zhou, W. Jiang, W. Zhou, X. Jing, J. Yu, B. Alkali and B. Sheng, "Analysis of Significant Factors on Cable Failure Using the Cox Proportional Hazard Model", IEEE Transactions on Power Delivery, vol. 29, no. 2, pp. 951-957, 2014.

[4] "IEEE Standard for the Testing, Design, Installation, and Maintenance of Electrical Resistance Trace Heating for Industrial Applications - Redline", IEEE, pp. 1-143, 2011.

[5] Z. Tang, C. Zhou, W. Jiang, W. Zhou, X. Jing, J. Yu, B. Alkali and B. Sheng, "Analysis of Significant Factors on Cable Failure Using the Cox Proportional Hazard Model", IEEE Transactions on Power Delivery, vol. 29, no. 2, pp. 951-957, 2014.

[6]X.dong, Y.yang, C.Zhou, "On-line Monitoring and Design of HV Cable Faults by Sheath System Currents", IEEE Transactions on Power Delivery, 2017. 